\title{
EFFICIENT SERIAL AND PARALLEL ALGORITHMS FOR MEDIAN FILTERING ${ }^{+}$ Sanjay Ranka* and Sartaj Sahni \\ University of Minnesota
}

\begin{abstract}
We develop a serial algorithm for separable median filtering that requires only two comparisons per element when the window size is three. In addition, fast parallel CREW PRAM algorithms with good processor-time product are developed for separable median filtering and two dimensional median filtering.
\end{abstract}

\section{Keywords and Phrases}

Median filtering, separable median filtering, complexity, CREW PRAM algorithms 


\section{INTRODUCTION}

Median filters are widely used for smoothing operations in signal, speech, and image processing ([TUKE76], [JAYA76]). This filtering operation is performed on an $N \times N$ image matrix $I[1 . . N, 1 . . N]$ using a $W \times W$ window where $W=2 w+1$ is an odd number. The result of median filtering is an $N \times N$ matrix MEDIAN2D defined as:

$\operatorname{MEDIAN} 2 D[i, j]=\operatorname{median}\{I[a, b] \mid \operatorname{nbhd}(a, i, w, N)$ and $n b h d(b, j, w, N)\}$

where

$n b h d(p, q, r, s)= \begin{cases}\text { true } & (p-q) \bmod s \leq r \text { or }(q-p) \bmod s \leq r \\ \text { false } & \text { otherwise }\end{cases}$

A straightforward serial computation of MEDIAN2D takes $O\left(N^{2} W^{2}\right)$ time. This is easily reduced to $O\left(N^{2} W \log W\right)$ by using balanced search trees. Huang, Yang, and Tang [HUAN79] have developed an $O\left(N^{2} W\right)$ algorithm for the case when the image values are in the range 0 through $K-1$ (i.e., there are $K$ gray levels). Narendra [NARE81] has introduced a related filtering operation, separable median filter, that can be computed in $O\left(N^{2} \log W\right)$ serial time (The algorithm presented in [NARE81] for this takes $O\left(N^{2} W\right)$ time. This was improved to $O\left(N^{2} \log W\right)$ by Basu and Brown [BASU87]). The separable median filter, SMEDIAN2D, is obtained by computing two one dimensional medians as below:

$\operatorname{MEDIAN} 1 D[i, j]=\operatorname{median}\{I[a, j] \mid \operatorname{nbhd}(a, i, w, N)\}$

$\operatorname{SMEDIAN} 2 D[i, j]=\operatorname{median}\{\operatorname{MEDIAN} 1 D[i, b] \mid \operatorname{nbhd}(b, j, w, N)\}$

In this formulation, row medians are computed first and then column medians. An alternate is to compute column medians first and then row medians. Changing the order in this way will generally lead to different results.

Basu and Brown [BASU87] also develop special purpose hardware for separable median filtering. Parallel algorithms to compute MEDIAN2D on a pyramid computer have been proposed in [TANI82] and [STOU83]. Note that by using the $O(\log \log M)$ time $M$ processor algorithm of

\footnotetext{
${ }^{+}$This research was supported in part by the National Science Foundation under grants DCR84-20935 and MIP 86-17374

* Professor Ranka's current address is CIS Dept, 313 Link Hall, Syracuse University, Syracuse, NY 13244.
} 
[AJTA86] to find the median of $m$ elements, we can calculate SMEDIAN2D and MEDIAN2D in $O(\log \log W)$ time using $O\left(N^{2} W\right)$ processors and $O\left(N^{2} W^{2}\right)$ processors respectively. The resulting processor-time products are $O\left(N^{2} W \log \log W\right)$ and $O\left(N^{2} W^{2} \log \log W\right)$. We develop algorithms which have good processor-time product ( i.e., within polylogarithmic factor of the optimal serial algorithm). The results obtained in this paper are summarized below:

(1) In Section 2, we present an algorithm to compute MEDIAN1D for the case $W=3$. This algorithm requires at most two comparisons to compute each element of MEDIAN1D. For the case $W=3$, Basu and Brown [BASU87] present an algorithm that requires at most 2.5 comparisons per element of MEDIAN1D.

(2) In Section 3, we show that $O(\log W)$ comparisons per element of MEDIAN1D is a lower bound under the decision tree model. Hence, the algorithm of [BASU87] is asymptotically optimal for this problem.

(3) Parallel CREW PRAM algorithms for the separable median filter problem are developed in Section 4. One of these computes MEDIAN1D and hence SMEDIAN2D in $O\left(\log ^{2} W \log \log W\right)$ time using $O\left(N^{2} /(\log W \log \log W)\right)$ processors. The processor time product for this algorithm is $O\left(N^{2} \log W\right)$ which by the result of Section 3 is optimal. The other algorithm developed in this section computes MEDIAN1D in $O\left(\log ^{2} W\right)$ time using $O\left(N^{2} \log W\right)$ processors. The processor-time product for this algorithm is $O\left(N^{2} \log ^{3} W\right)$ which is suboptimal.

(4) The technique used in Section 4 is extended in Section 5 to obtain an $O\left(\log ^{2} W\right)$ CREW PRAM algorithm to compute MEDIAN2D. This algorithm uses $O\left(N^{2} \log ^{2} W\right)$ processors. Notice that the processor-time product of this algorithm is $O\left(N^{2} \log ^{4} W\right)$ compared to $O\left(N^{2} W \log W\right)$ for the serial algorithm using balanced search trees. We can obtain a serial $O\left(N^{2} \log ^{4} W\right)$ algorithm for MEDIAN2D by simulating our parallel CREW PRAM algorithm. 


\section{COMPUTING MEDIAN1D SERIALLY}

Basu and Brown [BASU87] show that when $W=3$ MEDIAN1D can be computed using at most 2.5 comparisons per element of MEDIAN1D. Their algorithm is presented in Figure 1. For simplicity, this $\mathrm{C}$ code computes only median $1 d[1 . . N-2]$ for the $N$ pixel image image [0..N-1]. In iteration $i$ of the for loop, two median $1 d$ values (median $1 d[i+1]$ and median $1 d[i+2]$ ) are computed. First, in $(a, b)$ we save image $[i+1]$ and image $[i+2]$ in ascending order. Now, median $1 d[i+1]$ is the median of \{image $[i], a, b\}$ and median $1 d[i+2]$ is the median of \{image $[i+2], a, b\}$. Since $a, b$ are in ascending order, each median is computed using the respective if statement of Figure 1.

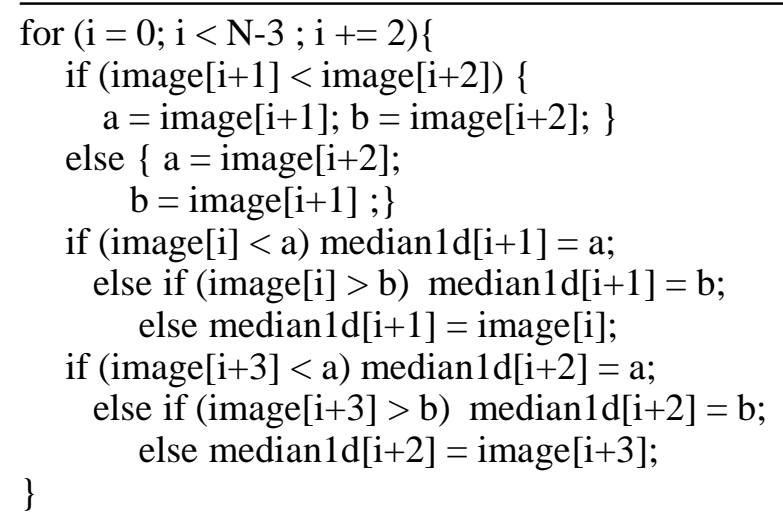

Figure 1: Basu and Brown's algorithm $(W=3)$

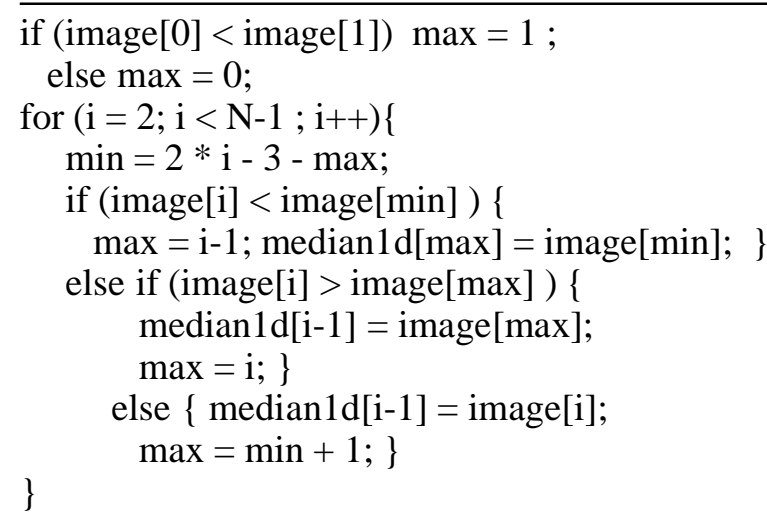

Figure 2: Finding the median $(W=3)$

In each iteration of the for loop of Figure 1, two median $1 d$ values are computed and at most 
5 comparisons are performed. Hence, at most 2.5 comparisons are performed per element of median $1 d$. On the average, however, each iteration requires only 13/3 comparisons (the probability of each image [] $>b$ compare being $2 / 3$ ) and the comparisons per element of median $1 d$ becomes 13/6. In Figure 2, we present an alternate way to compute median 1 d. Once again the code computes only median $1 d[1 . . N-2]$. median $1 d[i-1]=\operatorname{median}\{$ image $[i-2]$, image $[i-1]$, image $[i]\}, 1 \leq i \leq N-2$ At the start of each iteration of the for loop of Figure 2, max is such that image [max] is the larger of image $[i-2]$ and image $[i-1]$. The smaller of these two is given by the formula $2 i-3-$ max. The if statement in the for loop uses this information to compute median $1 d[i-1]$ and also to update max for the next iteration. The maximum number of comparisons per iteration of the for loop is 2 and the average (assuming a $2 / 3$ probability for the image [] > image [ $\max ]$ comparisons) is $5 / 3$.

The code of Figures 1 and 2 was run on an Apollo DN330 workstation. For worst case data

(image $[0 . . N-1]=(212243546 \ldots) \quad$ for $\quad$ Figure $\quad 1) \quad$ and (image $[0 . . N-1]=(1 N 2 N-13 N-24 N-3 \ldots)$.$) for Figure 2) and N=16024$, Figure 1 took 0.4 seconds while Figure 2 took 0.376 seconds. Hence our new algorithm takes $6 \%$ less time than the algorithm of Figure 1 on worst case data. The average run time of Figure 2 is about $2.4 \%$ less than that of Figure 1. While our new algorithm runs marginally faster than the algorithm of [BASU87], its main contribution is theoretical. It uses fewer comparisons.

\section{A LOWER BOUND FOR MEDIAN1D}

Theorem 1: For the decision tree model of computation ([HORO86], pp. 341), computing MEDIAN1D requires $\Omega(\log W)$ comparison, on average, per element in MEDIAN1D.

Proof: The proof is similar to that used by Shamos [SHAM76] to show that $\Omega(n \log n)$ is a lower bound for the online medians problem.

Let $A_{1} \cdots A_{3 w}$ be $3 \mathrm{w}$ independent sequences of $w$ distinct numbers each. Let $a$ and $b$ be such that $a$ is smaller than every number in the $A_{i}$ 's and $b$ is greater than every number in the $A_{i}$ 's. Construct the $N \times N, N=3 w$, image matrix I such that row $i$ is: 


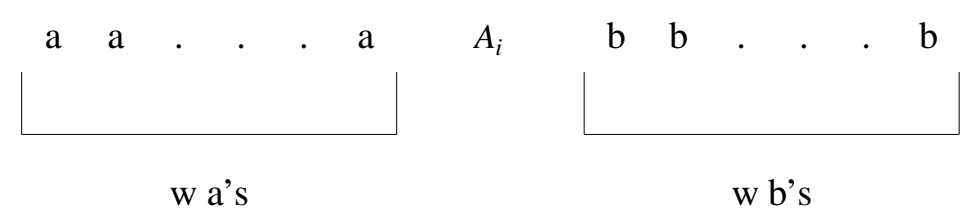

One may verify that the $i$ ' th row of MEDIAN1D with $W=2 w+1$ has the form:

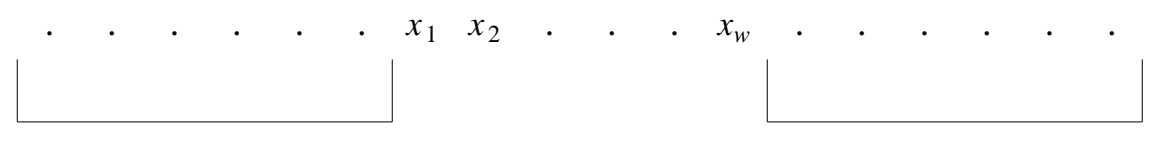

w values

w values

where $x_{1} \cdots x_{w}$ are the numbers of $A_{i}$ in ascending order. So, using this construction, we can use every algorithm for MEDIAN1D to sort $3 w$ sequences of size $w$. The number of different possible outcomes for such a sort is $(w !)^{3 w}$. Under the decision tree model [HORO86], every algorithm to sort these $3 w$ sequences must have average depth $\Omega\left(3 w^{2} \log w\right)$. Consequently, under this model, every algorithm to compute MEDIAN1D must have average complexity $\Omega\left(3 w^{2} \log w\right)$. So, $\Omega(\log w)=\Omega(\log W)$ work must be done, on average, per element of MEDIAN1D. This result can be easily extended to the case $N=6 w, 9 w, \ldots$

\section{CREW PRAM ALGORITHMS FOR SEPARABLE MEDIAN FILTER}

Suppose we have an $N \times N$ image $I$ and a $1 \times W$ window, $W=2 w+1$. The fastest way to compute MEDIAN1D on a CREW PRAM is to use $W$ processors per element of MEDIAN1D. Each group of $W$ processors finds $\operatorname{MEDIAN} 1 D[i, j]$ for a distinct index pair $(i, j)$. This is done in $O(\log \log W)$ time by using the algorithm of [AJTA86] to compute the median of the elements

$\{[[a, j] \mid \operatorname{nbhd}(a, i, w, N)\}$

The processor-time product for this algorithm is $O\left(N^{2} W \log \log W\right)$ which is not optimal. The processor-time product for the asymptotically optimal algorithm of [BASU87] is $O\left(N^{2} \log W\right)$. In this section, we develop two parallel algorithms for MEDIAN1D (and hence SMEDIAN2D) with processor time-products $O\left(N^{2} \log ^{3} W\right)$ and $O\left(N^{2} \log W\right)$ respectively. The first has a run time $O\left(\log ^{2} W\right)$ and uses $O\left(N^{2} \log W\right)$ processors. The run time of the second algorithm is 
$O\left(\log ^{2} W \log \log W\right)$ and the processor requirement is $O\left(N^{2} /(\log W \log \log W)\right)$. So, while the second algorithm has an optimal processor-time product, its run time is not optimal. Neither the run time nor the processor-time product of our first algorithm is optimal. However, the first algorithm is asymptotically faster then the second and serves as an introduction to the second. The existence of an $O(\log \log W)$ CREW PRAM algorithm with optimal processor-time product remains an open problem.

\subsection{First Algorithm}

In this algorithm, $\operatorname{MEDIAN} 1 D[i, j]$ is computed independently for each row $i$. Let $x_{1}, x_{2}, \cdots, x_{N}$ be the image values in some row $i$ of $I$. For simplicity, we assume that $w$ divides $N$ and $N / w>2$. Partition $x_{1}, \ldots, x_{N}$ into $N / w$ segments $S_{1}, S_{2}, \ldots, S_{N / w}$. This is done left to right with $x_{1}, \ldots, x_{w}$ in $S_{1} ; x_{w+1}, \ldots, x_{2 w}$ in $S_{2}$; etc. The steps in Algorithm 1 are:

Step 1: $\quad$ Let $Y_{a}=S_{(a-2) \bmod N / w+1} \cup S_{a} \cup S_{a \bmod N / w+1}, 1 \leq a \leq N / w$. Sort each $Y_{a}$.

Step2: $\quad$ Let $\operatorname{rank}(l e f t, j)=$ position of $x_{j}$ in $Y_{(a-2) \bmod N / w+1}$, $\operatorname{rank}($ middle,$j)=$ position of $x_{j}$ in $Y_{a}$, $\operatorname{rank}($ right,$j)=$ position of $x_{j}$ in $Y_{(a) \bmod N / w+1}$, where $a=\lceil j / w\rceil$

Step3: $\quad$ Sort each $S_{b}$ by repeatedly merging adjacent subsequences of size at most $2^{j}, 0 \leq j<\log _{2} w$. The merged subsequences for each $j$ are saved.

Step4: $\quad$ For each $l, 1 \leq l \leq N$ form the subsequence set, $Z_{l}$, (from the results of Step3) that consists of the $W=2 w+1$ elements $x_{c}, \operatorname{nbhd}(c, l, w, N)$.

Step5: $\quad$ Search $Z_{l}$ for the median element. This element is $\operatorname{MEDIAN} 1 D[i, l]$.

Consider the case $w=4, \quad$ and $\quad N=16$. Let $\left[x_{1}, \ldots, x_{16}\right]$ be $[2,4,6,1,3,9,8,7,11,14,13,5,10,12,16,15]$. For this, we get:

$$
\begin{aligned}
& Y_{1}=10,12,16,15,2,4,6,1,3,9,8,7 \\
& Y_{2}=2,4,6,1,3,9,8,7,11,14,13,5 \\
& Y_{3}=3,9,8,7,11,14,13,5,10,12,16,15
\end{aligned}
$$


$Y_{4}=11,14,13,5,10,12,16,15,2,4,6,1$

Let $S Y_{i}$ represent the sorted version of $Y_{i}$ following Step 1. We have:

$S Y_{1}=1,2,3,4,6,7,8,9,10,12,15,16$

$S Y_{2}=1,2,3,4,5,6,7,8,9,11,13,14$

$S Y_{3}=3,5,7,8,9,10,11,12,13,14,15,16$

$S Y_{4}=1,2,4,5,6,10,11,12,13,14,15,16$

The quadruples $\left(X_{i}, \operatorname{rank}(l e f t, i), \operatorname{rank}(\right.$ middle,$\left.i), \operatorname{rank}(\operatorname{right}, i)\right)$ for $1 \leq i \leq 16$ are:
$(1,1,1,1)$
$(5,5,2,4)$
$(9,8,9,5)$
$(13,11,9,9)$
$(2,2,2,2)$
$(6,5,5,6)$
$(10,6,6,9)$
$(14,12,10,10)$
$(3,3,3,1)$
$(7,6,7,3)$
$(11,10,7,7)$
$(15,11,11,11)$
$(4,3,4,4)$
$(8,7,8,4)$
$(12,8,8,10)$
$(16,12,12,12)$

The step 3 pairwise merging takes the form:

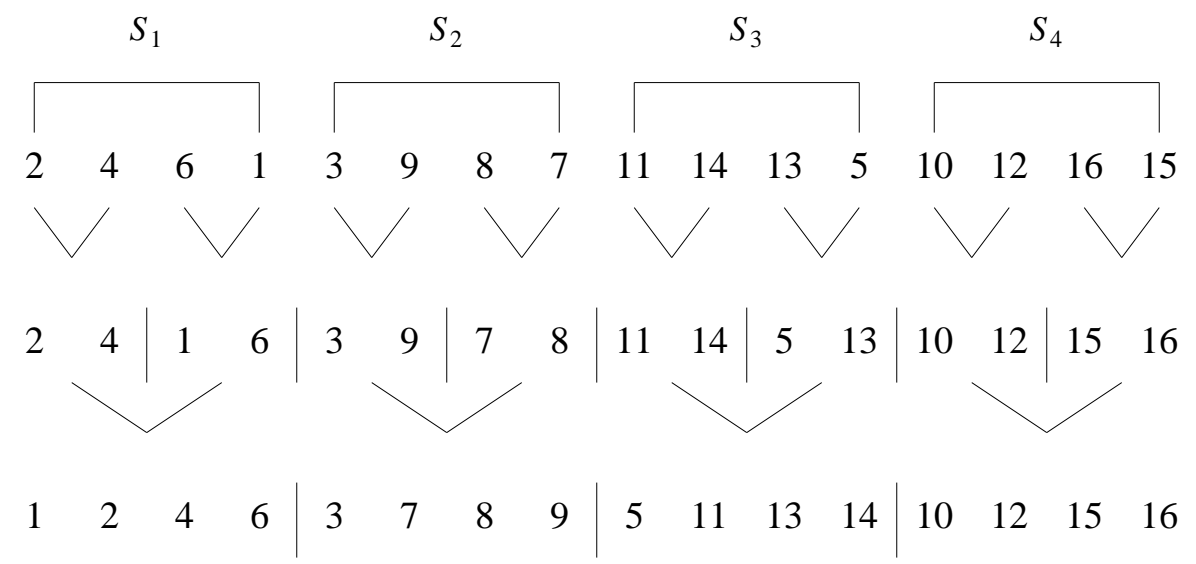

For step 4 , consider the case $l=6$. The elements $x_{l}$ are $\left\{x_{2}, x_{3}, x_{4}, x_{5}, x_{6}, x_{7}, x_{8}, x_{9}, x_{10}\right\}$.

The necessary sorted subsequences are:

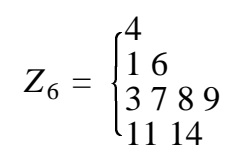

It is easy to see that $\left|Z_{l}\right|$ is $O(\log w)$.

To find the median of these $W=2 w+1$ elements we replace each $x_{i}$ in $Z_{l}$ by a pair $\left(x_{i}, r a n k_{i}\right)$ 


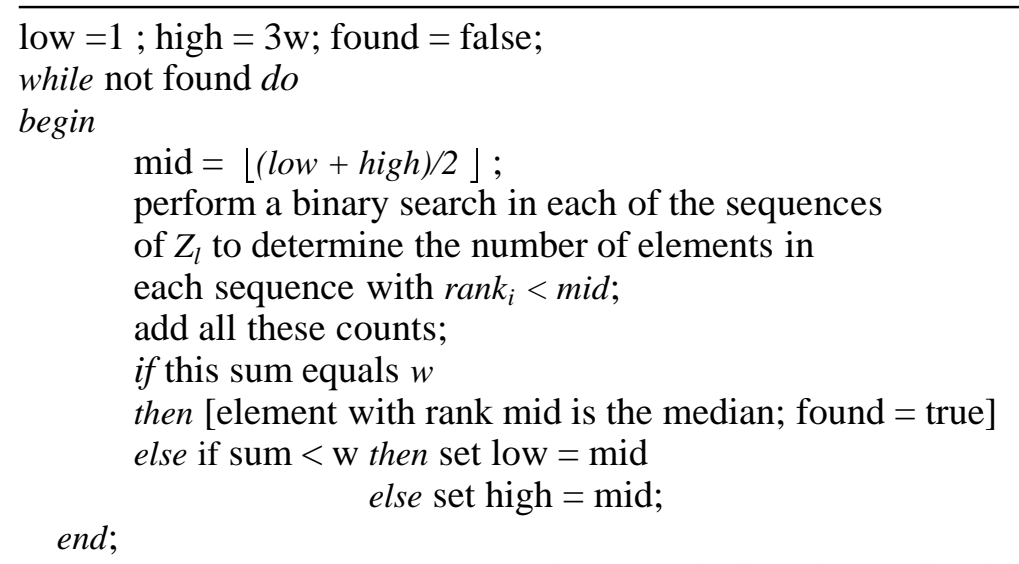

Figure 3: Finding the median in $Z_{l}$

where $\operatorname{rank}_{i}$ is as given below:

$$
\operatorname{rank}_{i}=\left\{\begin{array}{l}
\operatorname{rank}(\text { left, } i) \text { if } x_{i} \in S_{(a-2) \bmod N / w+1} \\
\operatorname{rank}\left(\text { middle, } \text { i) if } x_{i} \in S_{a}\right. \\
\operatorname{rank}(r i g h t, i) \text { if } x_{i} \in S_{a \text { mod } N / w+1}
\end{array}\right.
$$

where $a=\lceil l / w\rceil$. For our example, the transformed $Z_{6}$ is:

$$
\begin{aligned}
& (4,4) \\
& (1,1)(6,6) \\
& (3,3)(7,7)(8,8)(9,9)
\end{aligned}
$$$$
(11,10)(14,12)
$$

Note that since all the $x_{i}$ 's in $Z_{l}$ come from the same $Y_{a}$, the $r a n k_{i}$ values are distinct and in the range $[1,3 w]$. To find the element with median rank, we perform a binary search as in Figure 3. For our example, the while loop of Figure 3 is iterated 3 times. A trace of Figure 3 is given below:

$\begin{array}{ccccc}\text { iteration } & \text { low } & \text { high } & \text { mid } & \text { sum } \\ 1 & 1 & 12 & 6 & 3 \\ 2 & 6 & 12 & 9 & 6\end{array}$




$\begin{array}{lllll}3 & 6 & 9 & 7 & 4\end{array}$

On termination, mid $=7$ and the element with this rank is 7 . So, the median is 7 .

\section{Complexity Analysis}

On a CREW PRAM, each $Y_{a}$ can be formed in $O(1)$ time using $O(W)$ processors. So, all $Y_{a}$ 's can be formed in parallel in $O(1)$ time using $O(N)$ processors. All $Y_{a}$ 's can be sorted in $O\left(\log ^{2} W\right)$ time using $O(W)$ processors per $Y_{a}$ for a total of $O(N)$ processors. This can be done using a bitonic sort [BILA86]. Step 2 is easily performed in $O(1)$ time using $O(N)$ processors. Step 3 can be done in $O\left(\log ^{2} W\right)$ time using bitonic sort and $O(N)$ processors. The subsequence sets $Z_{l}$ can now be identified in $O(\log W)$ time using 1 processor for each $l, 1 \leq l \leq N$. To find the median of each $Z_{l}, O(\log W)$ iterations of the while loop of Figure 3 are to be performed. The binary search for each subsequence of $Z_{l}$ can be done in $O(\log W)$ time. So, if we have $O(\log W)$ processors assigned to each $Z_{l}$, each iteration of the while loop will take $O(\log W)$ time (as each subsequence of $Z_{l}$ is of size at most $W$ and $\left|Z_{l}\right|$ is $\left.O(\log W)\right)$. The total time for Step 4 is therefore $O\left(\log ^{2} W\right)$ when $O(N \log W)$ processors are available. The processor-time product is $O\left(N \log ^{3} W\right)$.

To compute the MEDIAN1D values for all $\mathrm{N}$ rows of $\mathrm{I}$ in parallel requires $\mathrm{N}$ times as many processors. The run time remains $O\left(\log ^{2} W\right)$ but the processor requirement becomes $O\left(N^{2} \log W\right)$. Once, MEDIAN1D has been computed, SMEDIAN2D may be computed by applying a similar algorithm using MEDIAN1D as the image. The run time, processor requirement, and processortime product are unchanged.

\subsection{Second Algorithm}

As in Algorithm 1, MEDIAN $1 D[i, j]$ is computed independently for each row $i$. The steps in Algorithm 2 are:

Steps1-3: $\quad$ Same as Steps 1-3 of algorithm 1.

Step 4: $\quad$ For $l=1, \log ^{2} w+1,2 \log ^{2} w+1, .$. form the subsequence set $Q_{l}$ that consists exactly of the $W=2 w+1$ elements $x_{c}, n b h d(c, l, w, N)$. 
Step 5: $\quad$ Use $Q_{l}$ to find $\operatorname{MEDIAN}[i, l+r], 0 \leq r<\log ^{2} w$

Consider the same example as for Algorithm 1. While, in Algorithm 1, $Z_{l}$ is formed for $1 \leq l \leq N=16$, in Algorithm 2, Only $Q_{1}, Q_{5}, Q_{9}$, and $Q_{13}$ are formed. $Q_{9}$, for instance, consists of elements $x_{5} \cdots x_{13}$. The subsequences used are slightly different from those used in $Z_{9}$. For $Q_{l}$, the subsequences obtained in Step 3 are used only to cover the last $W-\log ^{2} w$ elements of $Q_{l}$. The first $\log ^{2} w$ elements are covered by a new sequence obtained by sorting these $\log ^{2} w$ elements. So, $Q_{9}$ consists of the subsequences:

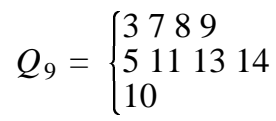

Let the subsequences in $Q_{l}$ be $A_{0}, A_{1}, \ldots ., A_{k}$ with $A_{0}$ being the sequence that contains the first $\log ^{2} w$ elements of $Q_{l}$. Let $A_{k+1}$ be another, initially empty, sequence. Since we shall be deleting from $A_{0}$ and inserting into $A_{k+1}$, we maintain these as balanced binary search trees. MEDIAN $1 d[i, l]$ is computed using the algorithm of Figure 3 modified to handle the search trees $A_{0}$ and $A_{k+1}$. In addition, the algorithm computes $S(i)$ and $B(i)$, where $S(i)$ is the largest element in $A_{i}$ smaller than the median of $Q_{l}$ and $B(i)$ is the smallest element in $A_{i}$ bigger than this median. $S(i)$ and $B(i)$ are null if $A_{i}$ does not contain such elements.

For $Q_{9}$ of our example, the median, $\operatorname{MEDIAN}(i, 9)$, is 9. So, $S(0)=8, S(1)=5, S(2)=p h i, S(3)=\phi, B(0)=\phi, B(1)=11, B(2)=10, \quad$ and $\quad B(3)=\phi . \quad$ Now $\operatorname{MEDIAN} 1 D[i, r]$ for $r=10,11$ and 12 are computed by successively forming $Q_{10}, Q_{11}$, and $Q_{12}$. For $Q_{10}$, we add $x_{14}=12$ to $A_{3}$ and delete $x_{5}=3$ from $A_{0}$ to get:

$$
\begin{aligned}
& A_{0}=789 \\
& A_{1}=5111314 \\
& A_{2}=10 \\
& A_{3}=13
\end{aligned}
$$

This requires us to update $B(3)$ to 12 . There are four cases in the computation of the new median:

Case 1: The element deleted from $A_{0}$ as well as that added to $A_{k+1}$ are smaller than the previous median. In this case the median is unchanged. 
Case 2: The element deleted from $A_{0}$ as well as that added to $A_{k+1}$ are bigger than the previous median. Here too, the median is unchanged.

Case 3: The element deleted from $A_{0}$ is less than or equal to the previous median but that added to $A_{k+1}$ is bigger. The new median is $\min \{B(s) \mid 0 \leq s \leq k+1$ and $B(s) \neq \phi\}$.

Case 4: The element deleted from $A_{0}$ is greater than or equal to the previous median while that added to $A_{k+1}$ is smaller. The new median is $\max \{S(s) \mid 0 \leq s \leq k+1$ and $S(s) \neq \phi\}$.

For our example, the deleted element is 3 and the inserted element 12. Since the previous median was 9, we are in Case 3 and the new median is $\min \{11,10,12\}=10$. The S's and B's are now updated to:

$$
\begin{array}{ll}
S(0)=9 & B(0)=\phi \\
S(1)=5 & B(1)=11 \\
S(2)=\phi & B(2)=\phi \\
S(3)=\phi & B(3)=12
\end{array}
$$

For MEDIAN $1 D[i, 11]$ we delete $x_{6}=9$ and insert $x_{15}=16$ to get:

$$
\begin{aligned}
& A_{0}=78 \\
& A_{1}=5111314 \\
& A_{2}=10 \\
& A_{3}=1216
\end{aligned}
$$

This causes $B(0)$ to be updated to 8 . Since the inserted element is bigger than the previous median and the deleted element smaller, we are again in Case 3. The new median, $\operatorname{MEDIAN} 1 D[i, 11]$, is $\min \{11,12\}$. The S's and B's are updated and we proceed to compute MEDIAN $1 D[i, 13]$ by first deleting $x_{7}$ from $A_{0}$ and inserting $x_{16}$ into $A_{3}$.

\section{Complexity Analysis}

For this, we shall assume that only $O(N /(\log W \log \log W))$ processors are available. Using the bitonic sort algorithm of Bilardi and Nicolau [BILA86], Steps 1 through 3 can be done on a CREW PRAM in $O\left(\log ^{2} W \log \log W\right)$ time. For Step 4, we note that $O\left(N / \log ^{2} w\right) Q_{l}$ 's are to be formed. Thus, for each we have $O(\log W / \log \log W)$ processors. Since $A_{0}$ initially contains $\log ^{2} W$ 
elements, the height of its balanced tree representation is $O(\log \log W) . A_{0}$ may be set up in $O\left(\log ^{2} W \log \log W\right)$ time using one processor. The remaining $A_{i}$ 's are easily accumulated in $O(\log W)$ time using a single processor. The algorithm of Figure 3 takes $O\left(\log ^{2} W \log \log W\right)$ time to find the median of a $Q_{l}$ when $O(\log W / \log \log W)$ processors are available to each $Q_{l}$. The B's and S's are a byproduct of the searches used to find the median and may now be initialized in $O(\log \log W)$ time. For the remaining medians, each insertion into $A_{k+1}$ and each deletion from $A_{0}$ can be done in $O(\log \log W)$ time with a single processor. All the B's and the S's may also be updated in this much time using $O(\log W / \log \log W)$ processors. The $\min$ of the $O(\log W) \mathrm{B}$ 's or the max of the $O(\log W)$ S's can also be computed in $O(\log \log W)$ time using $O(\log W / \log \log W)$ processors. So the computation of the remaining $\log ^{2} W-1$ medians for each $Q_{l}$ takes $O\left(\log ^{2} W \log \log W\right)$ time. Hence, one row of MEDIAN1D can be computed in $O\left(\log ^{2} W \log \log W\right)$ time using $O(N /(\log W \log \log W))$ processors. All $\mathrm{N}$ rows can be computed in this much time using $O\left(N^{2} /(\log W \log \log W)\right)$ processors. This algorithm has an optimal processor-time product of $O\left(N^{2} \log W\right)$.

\section{TWO DIMENSIONAL MEDIAN FILTERING}

Our parallel algorithm for MEDIAN2D is an adaptation of Algorithm 1 for MEDIAN1D. The Steps are:

Step1: $\quad$ Partition the $N \times N$ image into $w \times w$ subimages $S_{i, j}$ as in Figure 3. For each $S_{i, j}$ form $Y_{i, j}=\underset{\begin{array}{c}n b h d(i, k, 1, N / w) \\ n b h d(j, l, 1, N / w)\end{array}}{\cup} S_{k, l}$ and sort $Y_{i j}, 1 \leq i, j \leq N / w$.

Step2: $\quad$ Rank the elements of the $N \times N$ image $I$. Each element $I[a, b]$ will have nine ranks $\operatorname{rank}(y, z, a, b), y \in\{$ top row, middle row, bottom row $\}, z \in\{$ left column, middle column, right column $\}$. Let $S_{c, d}$ be the subimage that contains $I[a, b] \cdot \operatorname{rank}(y, z, a, b)$ is obtained by looking at each of the nine $Y_{i, j}$ 's that contain $S_{c, d} \cdot y$ and $z$ are defined by the position of $S_{c, d}$ in $Y_{i, j}$ (see Figure 3) and rank is the position of $I[a, b]$ in the sorted $Y_{i, j}$.

Step3: $\quad$ Each $S_{i, j}$ may be tiled with rectangles of size $2^{f} \times 2^{g}, 0 \leq f, g \leq\left\lfloor\log _{2} w\right\rfloor$. When $w$ is 


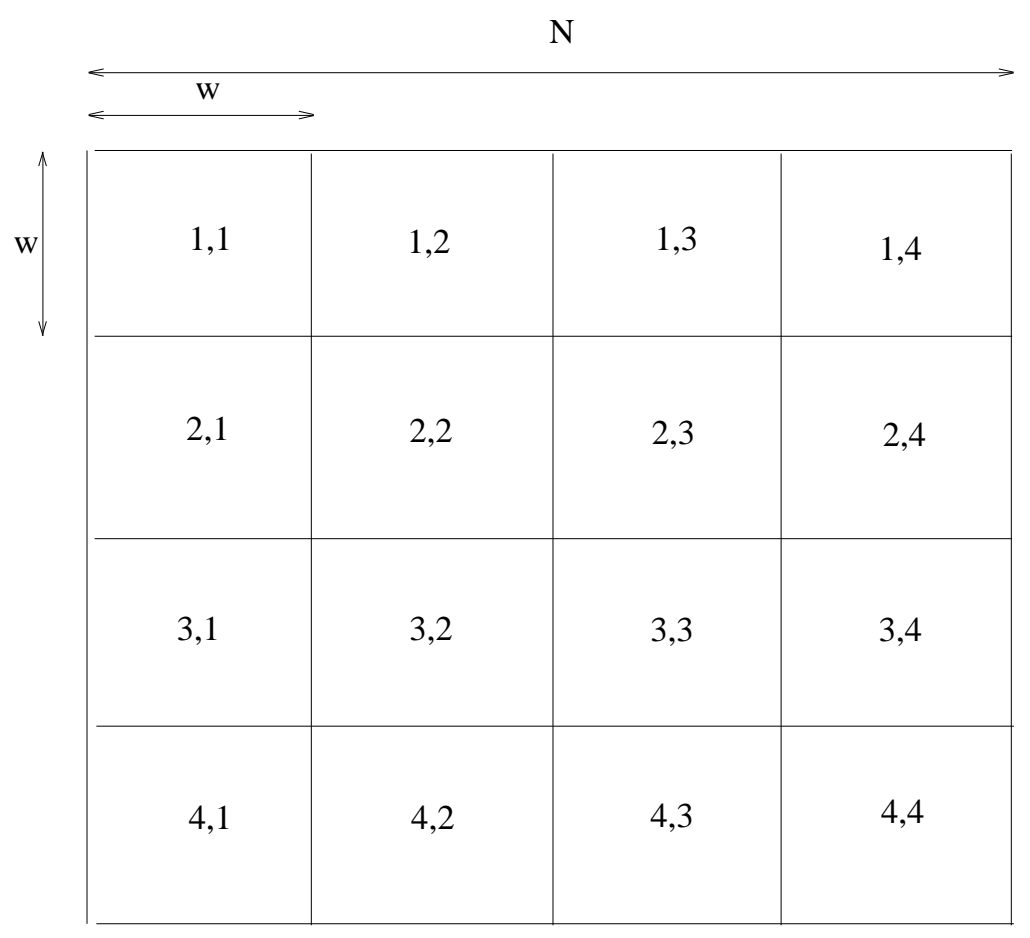

Figure 3: Partitioning of $N \times N$ image into $w \times w$ subimages

not a power of 2, some partial tiles may be used at the ends. Figure 5 shows the tiling for the case $w=7$.

Step4: $\quad$ For each pair $(i, j), 1 \leq i, j \leq N$ form the sorted sequence set, $U_{i, j}$, from Step 3 that includes exactly the elements $\{I[a, b] \mid \operatorname{nbhd}(i, a, w, N)$ and $n b h d(j, b, w, N)\}$

Step5: $\quad$ Search $U_{i, j}$ for the median element. This is $\operatorname{MEDIAN} 2 D[i, j]$.

\section{Complexity Analysis}

For the analysis, we assume that $O\left(N^{2} \log ^{2} W\right)$ processors are available. Each $Y_{i j}$ of step 1 has $O\left(W^{2}\right)$ elements. As there are $N^{2} / W^{2} Y_{i j}$, s, we can allocate $O\left(W^{2}\right)$ processors to sort each. This sort can be done in $O\left(\log ^{2} W\right)$ time with this many processors. The ranking of step 2 can now be done in $O$ (1) time. The step 3 sorting may be done by repeatedly merging smaller tiles to get bigger ones. In each merge stage pairs of tiles of some fixed size are merged to get sorted tiles of the next size. There are $O\left(\log ^{2} W\right)$ tile sizes and $O(\log W)$ merge stages each taking $O(\log W)$ time are needed (Figure 6). So, step 3 is done in $O\left(\log ^{2} W\right)$ time with the given number of processors. 


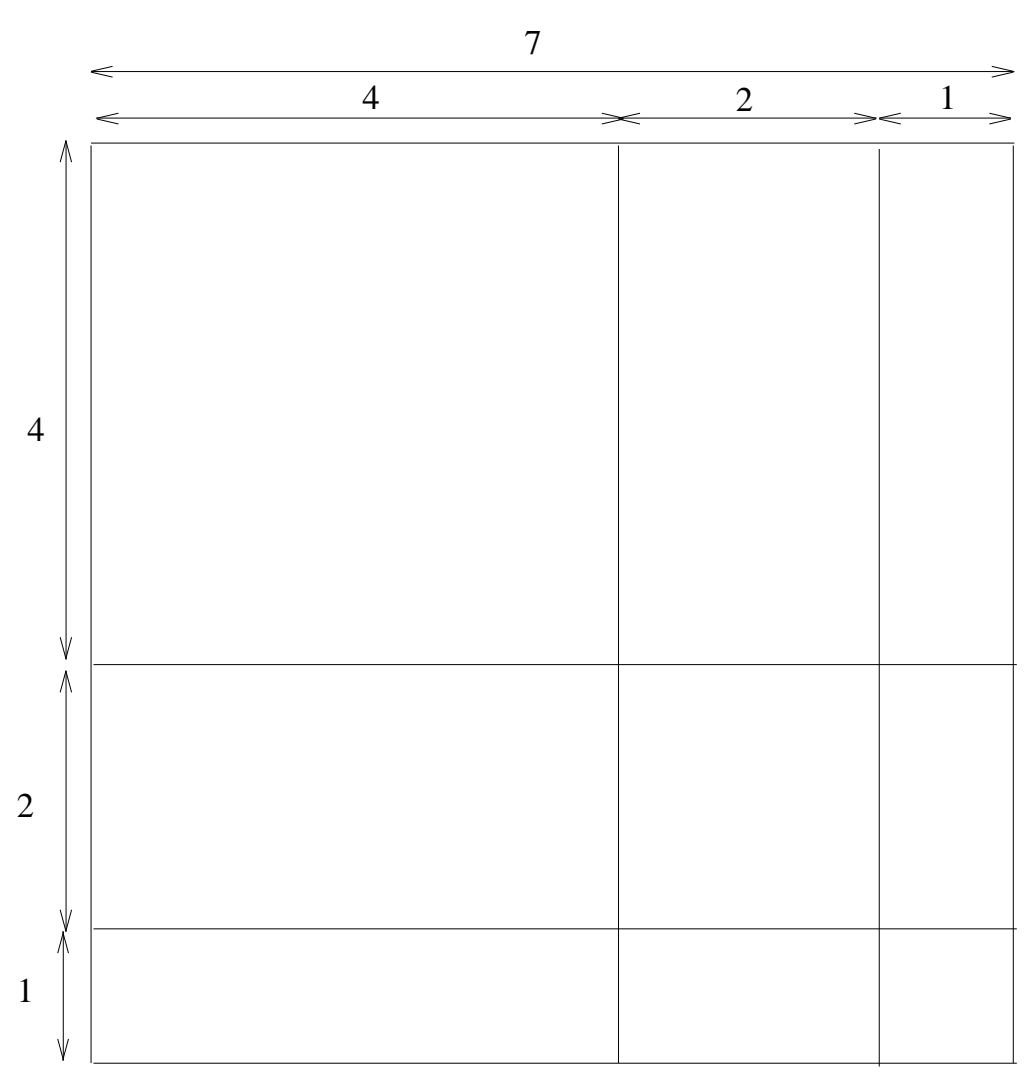

Figure 5: Tiling of $S_{i, j}(w=7)$

For step 4, 1 processor is used to form each $U_{i j}$. Each $U_{i j}$ can be formed so as to have only $O\left(\log ^{2} W\right)$ sorted sequences. Each such sequence is at most $O\left(W^{2}\right)$ long. The step 4 time is $O\left(\log ^{2} W\right)$. The step 5 search of each $U_{i j}$ is done using an algorithm almost identical to that of Figure 3 (the rank range needs to be increased from $3 w$ to $\left.9 w^{2}\right)$. Using $O\left(\log ^{2} W\right.$ ) processors for each $U_{i j}$, this search takes $O\left(\log ^{2} W\right)$ time. So the total time for the algorithm is $O\left(\log ^{2} W\right)$ and the processor requirement is $O\left(N^{2} \log ^{2} W\right)$. The processor-time product is $O\left(N^{2} \log ^{4} W\right)$ which is better than that for the serial algorithm that uses balanced search trees.

\section{CONCLUSION}

We have developed an algorithm for separable median filtering that requires at most 2 comparisons per median element when the window size is 3 . This represents a theoretical improve- 


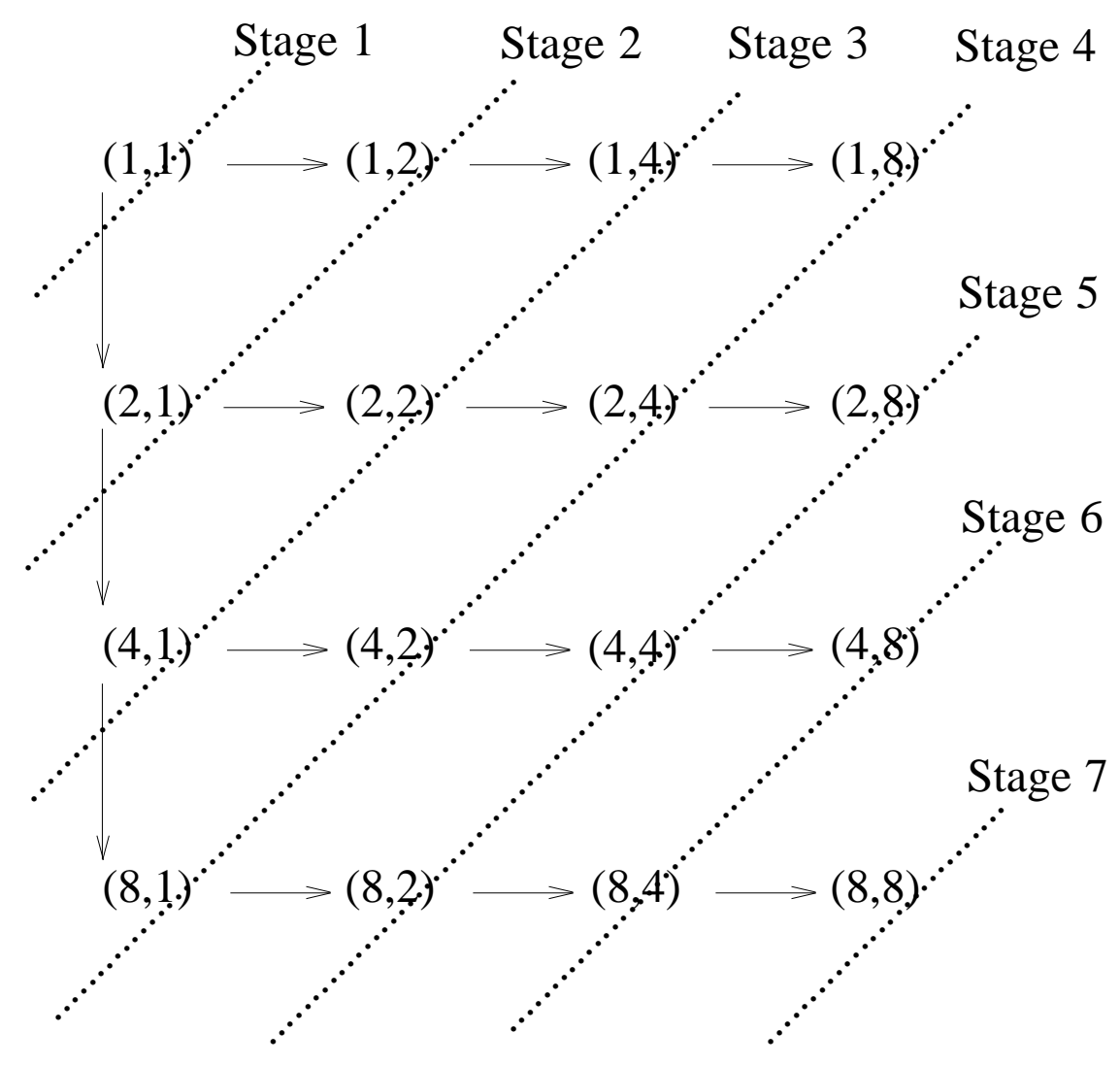

... represent the stages

---> represent the dependencies

Figure 6: Stages required in Merging

ment over the result of [BASU87]. Our algorithm, however, runs only marginally faster that of [BASU87]. Fast parallel CREW PRAM algorithms with good processor-time product have also been developed for separable median filtering and two dimensional median filtering. The following problems remain open:

(1) Is there an $O(\log N)$ CREW PRAM algorithm with optimal processor-time product for Separable Median Filtering?

(2) What is the complexity of an optimal serial algorithm for two dimensional median filtering?

(3) Find an $O(\log N)$ CREW PRAM algorithm for two dimensional median filtering with good processor-time product (within a polylogarithmic factor of the optimal). 


\section{REFERENCES}

[AJTA86] M. Ajtai, J. Komlos, W. L. Steiger, and E. Szemeredi, "Deterministic Selection in $O(\log \log N)$ Parallel Time", Proceedings of 18th ACM Symposium on Theory of Computing, 1986, pp. 188-195.

[BALL85] D. H. Ballard and C. M. Brown, "Computer Vision", 1985, Prentice Hall, New Jersey.

[BASU87] A. Basu and C. M. Brown,"Algorithms and Hardware for Efficient Image Smoothing", Computer Vision, Graphics and Image Processing", Vol. 40, February 1987, pp. 131-146.

[BILA86] G. Bilardi and A. Nicolau, "Bitonic sorting with $O(N \log N)$ comparisons", Proceedings of 1986 Conference on Information and System Sciences, Department of Electrical Engineering, Princeton University, pp. 366-341.

[JAYA76] N. S. Jayant, "Average and median based smoothing techniques for improving digital speech quality in the presence of transmissions error", IEEE Trans. on Communication, Vol. COM-24, September 1976, pp. 1043-1049.

[HORO86] E. Horowitz and S. Sahni, "Fundamentals of Data Structures in Pascal", Computer Science Press, Maryland, 1986.

[HUAN79] T. S. Huang, G.Y. Yang, and G. Y. Tang, "A Fast Two dimensional Median Filtering Algorithm", IEEE Transaction on ASSP, Vol. ASSP-27, No. 1, February 1979, pp. 13-18.

[NARE81] P.M. Narendra. "A Separable Median Filter for Image Noise Smoothing", IEEE Trans. on PAMI, Vol. PAMI-3, No. 1, January 81, pp. 20-29.

[RABI75] L. R. Rabiner, M. R. Sanbur and C. E. Schmidt, "Applications of a non-linear smoothing operation to speech processing," IEEE Transaction on ASSP, Vol. ASSP-23, December 1975, pp. 552-557.

[SHAM76] M. I. Shamos, "Geometry and Statistics: Problems at the Interface", Algorithms and 
Complexity: New Directions and Recent Results, Edited by J.F. Traub, Academic Press Inc., New York, 1976.

[STOU83] Q. Stout, "Sorting, Merging, Selecting and Filtering on Tree and Pyramid Machines", Proceedings of 1983 International Conference on Parallel Processing, pp. 214-221.

[TANI82] S. L. Tanimoto, "Sorting, histogramming, and other statistical operations on a pyramid machine", Department of Computer Science Report 82-08-82, University of Washington, Seattle. 